\title{
sciendo
}

\section{Comparison of the Effects of Three Hangboard Strength and Endurance Training Programs on Grip Endurance in Sport Climbers}

\author{
by \\ Eva López-Rivera ${ }^{1}$, Juan José González-Badillo²
}

\begin{abstract}
Intermittent isometric endurance of the forearm flexors is a determinant factor of sport climbing performance. However, little is known about the best method to improve grip endurance in sport climbing regarding maximal or intermittent dead-hang training methods. The aim of this study was to compare the effects of three 8-week finger training programs using dead-hangs (maximal, intermittent, and a combination) on grip endurance. Twenty-six advanced sport climbers $(7 \mathrm{c}+/ 8$ a mean climbing ability) were randomly distributed among three groups: maximal deadhangs with maximal added weight on an $18 \mathrm{~mm}$ edge followed by MaxHangs on minimal edge depth; intermittent dead-hangs using the minimal edge depth, and a combination of both. The grip endurance gains and effect size were $34 \%$ and 0.6 , respectively, for the group following maximal dead-hang training, $45 \%$ and 1 , respectively, for the group following intermittent dead-hang training, and 7\% and 0.1, respectively, for the group applying the combination of both training methods. Grip endurance increased significantly after 4 weeks in the group performing intermittent deadhangs ( $p=0.004)$ and after 8 weeks in both groups performing intermittent dead-hangs $(p=0.002)$ and MaxHangs $(p=$ 0.010). The results suggest that the intermittent dead-hangs training method seems to be more effective for grip endurance development after eight week application in advanced sport-climbers. However, both methods, maximal and intermittent dead-hangs, could be alternated for longer training periods.
\end{abstract}

Key words: rock climbing, dead-hang training, intermittent isometric training, strength, endurance, climbing performance.

\section{Introduction}

The growing interest in climbing, as well as the participation at both recreational and competitive levels in the last decade has recently culminated with the inclusion of sport climbing in the Tokyo 2020 Olympic schedule. However, scientific research focused on optimal training programs and methodology for improving sport performance is still scarce.

The importance of intermittent isometric endurance of the forearm flexors for performance in climbing is generally acknowledged by climbers and several authors have pointed to it as a determinant factor for performance (Ferguson and Brown, 1997; Fryer et al., 2014; MacLeod et al.,
2007; Magiera et al., 2013; Philippe et al., 2012). Redpointing a rock climbing route (after having practiced the route beforehand) usually takes from 8 to 20 minutes, but can last twice or more for specific routes or onsight climbing. Fatigue during this time is explained by the succession of intermittent isometric contractions of relatively small muscles (MacLeod et al., 2007) and by the disproportionately short pauses between contractions (MacLeod et al., 2007; Philippe et al., 2012). Recently, it was reported that the forearm flexor oxidative capacity index is an important determinant of rock-climbing performance (Fryer et al., 2016).

1 - Faculty of Sport Sciences, Castilla La-Mancha University, Toledo, Spain.

2 - Faculty of Sport Sciences, Pablo de Olavide University, Seville, Spain. 
The more intense sections that dictate the difficulty of a climb tend to include poorly shaped (like slopers) or smaller handholds than the rest (Amca et al., 2012), specifically one phalange deep or less (Schweizer and Hudek, 2011; Vigouroux, 2006), or frequently farther apart or on steeper walls, requiring in all cases a high percentage of the finger flexors' maximum strength (Watts, 2004). The positive effect of using extra weight in strength training is well known, especially with loads over $80 \%$ in experienced athletes (Hickson et al., 1988) or loads compatible with effort duration of 3 to $10 \mathrm{~s}$ (Pucci et al., 2006). Additionally, several authors have observed that strength training promotes endurance (Hickson et al., 1988; Marcinik et al., 1991; Østerås et al., 2002) as long as specific exercises are used (Tanaka et al., 1993). Hanging off an edge with the fingertips (dead-hangs) (López-Rivera and GonzálezBadillo, 2012; Watts, 2004) from a fingerboard or hangboard device is a specific exercise extensively used by climbers. To the best of our knowledge, there is only one controlled research that has tested the effect of fingerboard training (Medernach et al., 2015), although compared to the effect of bouldering training (modality consisting in climbing short and powerful routes, around $4 \mathrm{~m}$ high, with no rope and using landing mats for protection), not assessing different fingerboard programs. López-Rivera and González-Badillo (2012) tested fingerboard training methods using maximal strength training methods (MaxHangs), but not grip endurance methods. The best results were found with the program consisting of 4 weeks of MaxHangs using maximal added weight on a medium-depth edge (18 $\mathrm{mm}$, equivalent to one phalange), followed by 4 weeks of MaxHangs on minimal edge depth without added weight. Interestingly, increases in grip strength paired with increases in grip endurance were also found.

It has been proposed that high-volume, sub-maximal, short-recovery training promotes local muscle endurance (Ebben et al., 2004; McDonagh and Davies, 1984; Usaj, 2001). Still, for developing specific endurance it is important to use exercises (Usaj, 2001) and an exertion to recovery ratio typical of the sport (White and Olsen, 2010; Usaj, 2001), like cluster training (Iglesias et al., 2010) or intermittent training (Tomlin et al., 2001). In this line, intermittent dead-hangs (IntHangs), commonly known as "repeaters", constitute a popular training method to increase grip endurance in climbers. Medernach et al. (2015) examined training effects of 4 weeks of IntHangs on grip strength and endurance in competitive bouldering athletes. However, to our knowledge, there is a lack of research to date that has compared the effects of different strength and endurance methods using dead-hangs on grip endurance in climbing.

Thus, the aim of the current study was to test which intervention using the dead-hang exercise had the greatest influence on grip endurance: a) the most effective program in terms of grip strength and endurance in the study by López-Rivera and González-Badillo (2012) consisting of 8 weeks of MaxHangs; b) MaxHangs with maximal added weight for the first 4 weeks and then 4 weeks of intermittent dead-hangs (Max_IntHangs), or c) 8 weeks of intermittent dead-hangs (IntHangs). As a secondary objective, we intended to determine the existence of a relationship between maximum grip strength and grip endurance.

\section{Methods}

\section{Participants}

Thirty-eight (32 male and 6 female) welltrained sport climbers (French 7c+/8a, YDS: 5.13a redpoint climbing) who voluntarily signed up for the study, were recruited from the local climbing community following the previously set requisites of a) having at least 2 years of climbing experience, b) the minimum climbing level of $7 a$ according to the French scale (ranging from grade 5 to $9 b+$ ), c) being over 25 years old, d) not having used dead-hangs in the 4 months preceding the study, and e) no injury or condition that made inadvisable to undergo intense physical training. Their mean $( \pm \mathrm{SD})$ age was $31.7 \pm 6$ years; body mass: $65.61 \pm 8.57 \mathrm{~kg}$; body height: $171.66 \pm 7.43$ $\mathrm{cm}$. Twelve participants dropped out of the study ( 9 male and 3 female) due to illness, personal issues or other commitments, thus 26 participants (23 male and 3 female) completed the training program and were included for analysis.

Once the Ethics Committee (conforming to the Declaration of Helsinki) granted its approval, the participants were individually informed of the goals, procedures and potential risks deriving from the study, signed the 
informed consent and filled out a form with their contact details, age, climbing experience, training experience, and climbing ability. The participants were informed of the importance of observing consistent daily habits for the duration of the study. To aid the statistical analysis of the climbing ability data, the International Rock Climbing Research Association (IRCRA) scale proposed by Draper et al. (2015) for the conversion of climbing grades from French scale to a specific numerical values was used.

\section{Measures and Procedures}

This study was randomized, controlled and designed with the aim of comparing the effects of three different grip strength and grip endurance training programs using the deadhang exercise on grip endurance. In the MaxHangs method with added weight, the load was defined by the amount of extra weight attached to a belt that each participant wore, and in both the MaxHangs on minimal edge depth and IntHangs by the edge depth. Among the possible grip types, the half-crimp type was chosen for testing as well as for training given that it is the most widely used grip on small edges in climbing (Schweizer, 2001) and is safer than the full crimp. The edge depths used for the tests and for the added weight method were based on the criteria that the $15 \mathrm{~mm}$ edge depth (less than one phalange deep) was considered to be the most representative of the average size of holds used in competitions and existing in hard sections of climbing routes, and to have a high significant relationship with the climbing grade $(\mathrm{r}=0.81 ; p<$ $0.001)$. With this size as a basis, a bigger depth (18 $\mathrm{mm}$ ) was chosen for weekly training when following the MaxHangs with added weight method and a smaller one $(11 \mathrm{~mm})$ for the endurance test, given that according to Bourne et al. (2011), holding force for very small holds (smaller than $5 \mathrm{~mm}$ ) depends on finger anatomy (soft tissue) rather than muscular strength.

In week 0 , the initial grip strength and endurance tests (ST, ET, respectively) were conducted. The results were used to assign each participant to one of the three groups using the ABCCBA assignment procedure: the first individual was randomly chosen and included in group A and the BCCBA order was followed until all groups were complete. During training phase 1 (weeks 1 to 4), each group trained using one of the three prescribed methods and in phase 2 (weeks 5 to 8) the method was changed. On Monday of week 5 , strength and endurance tests were carried out (ST2 and ET2) to assess the effects of training during phase 1 , and the prescribed training was resumed the next day. On Monday of week 9 a new set of strength and endurance tests was conducted to measure the effects of training phase 2 (ST3 and ET3). Figure 1 provides an outline of the testing and training programs for clarity. Anthropometric data, climbing ability and characteristics of the groups are presented in Table 1.

The testing and training devices were those proposed by López-Rivera and GonzálezBadillo (2012). They consisted of a wooden edge of which depth could be varied with a precision within tenths of a millimeter. Two Casio brand chronometers were used to measure dead-hang duration, and Camp brand magnesium carbonate was used to reduce the effects of sweat.

The training session 48 hours before the tests was of low intensity and physical activity was avoided 24 hours before the tests. Each participant knew in advance the conditions and rules for the tests, and was already familiar with the correct dead-hang technique. The tests were carried out on Mondays at the same time of day, under similar conditions of relative humidity and temperature.

The warm-up routine before the test started with general mobilisation of the neck, shoulders, arms, wrists, and fingers, continuing with a specific segment of 3-5 sets of dead-hangs on a medium edge depth (15-20 $\mathrm{mm})$, progressing from 10 to $20 \mathrm{~s}$. Body mass and height were measured during the 5-min recovery interval between the warm-up and the first test. Then the maximum strength test was carried out, followed by the endurance test $10 \mathrm{~min}$ later.

Endurance test (ET) and Strength Test (ST). Both were conducted as proposed by López-Rivera and González-Badillo (2012), who performed a reliability study. We performed a validity analysis correlating the climbing level with test results, showing that the best climbers performed best on both initial tests $(r=0.51 ; p<0.001$ for ST and climbing level; $\mathrm{r}=0.62 ; p=0.001$ for ET and climbing level). The ET consisted of hanging off an $11 \mathrm{~mm}$-deep edge without added weight, to failure; defined as losing contact of the fingers 
with the edge, or flexing the arms, hips or extending the shoulders (Figure 2). The ST measured the maximum added weight that the athlete could hold for $5 \mathrm{~s}$, with straight arms, on a $15 \mathrm{~mm}$-deep edge. The method to determine the amount of added weight was as follows: an initial load was estimated, that would allow the participant to hang for $15-20 \mathrm{~s}$, and a $5 \mathrm{~s}$ deadhang was executed. After a 5-min pause, and according to the performance observed in the previous attempt, 5 to $10 \mathrm{~kg}$ were added. In order to prevent the effects of fatigue, the aim was to reach the maximum load in 5 attempts. When the participant could not hold the load for $5 \mathrm{~s}$, did not keep all four fingers of each hand in contact with the edge, or started flexing their arms, hips or extending their shoulders, the test was terminated, and the last valid load was recorded.

The participants received instruction on session load management and they were under supervision considering this effect during training. Dead-hang sessions were performed twice a week, on Mondays and Wednesdays, for two 4-week cycles. The general warm-up routine was the same used for testing; the specific segment consisted of 3-5 sets of $10 \mathrm{~s}$ dead-hangs, with progressively added weight or progressively smaller edges in MaxHangs, until the load for the session was reached, as detailed in López-Rivera and González-Badillo (2012). The specific IntHangs warm-up consisted of 4-5 repetitions of $10 \mathrm{~s}$ dead-hangs on progressively smaller edges, with $5 \mathrm{~s}$ pauses in-between.

Maximal dead-hangs (MaxHangs). The maximal strength training method with both maximum added weight and minimum edge depth were as described in López-Rivera and González-Badillo (2012). Each set involved hanging for $10 \mathrm{~s}$ with such a load (in terms of extra weight or edge depth, respectively) that allowed a potential hang time of $13 \mathrm{~s}$, leaving a three-second margin before failure. Extra weight and edge size for the first training session were determined as detailed in López-Rivera and González-Badillo (2012). This training program consisted of 3 to 5 sets of $10 \mathrm{~s}$ dead-hangs with $3 \mathrm{~min}$ rest between sets (three sets for week 1 and 5; four sets for week 2 and 6 , and five sets for week 3, 4, 7 and 8).

Intermittent dead-hangs (IntHangs). It consisted in hanging off an edge deep enough that allowed completing all the prescribed repetitions. The goal was to achieve failure or near failure in the last repetition of the last set, changing the edge depth between sets or repetitions when necessary. Edge size for the first training session was determined in advance, being one that would permit the participant to hang for around $30 \mathrm{~s}$. The $10 \mathrm{~s}$ contraction time was chosen because it matched the time per repetition in the strength methods, close to the mean grip duration during intense climbing sessions (White and Olsen, 2010). Each set was composed of 4-5 repetitions, adding up to $40-50$ s per set, like the average duration of the more intense segments in a route (estimated from video analysis and direct observation of 40 climbs from $8 b$ to $9 a$ ); such volume is equivalent to loads from 60 to $80 \%$ of maximal voluntary contraction, an intensity level compatible with positive effects on high intensity local endurance. The pause between repetitions was $5 \mathrm{~s}$, in line with the observed minimum time of 3-5 s for oxygenation between isometric, high-intensity intermittent contractions (Demura and Nakada, 2009; Fryer et al., 2014). This dead-hang program consisted of 3 to 5 sets of 4 ten-second repetitions each with $5 \mathrm{~s}$ rest between repetitions and $1 \mathrm{~min}$ rest between sets from week 1 to 4 . The group using this method for 8 weeks, during training phase 2 performed 5 repetitions instead, with the number of sets 3 for week 1 and 5 , four for week 2 and 6 and five sets for week $3,4,7$ and 8 .

Training sessions. The participants not only trained dead hangs; this workout was incorporated into regular climbing training sessions. One typical climbing session included 3 to 10 sets of 3 to 90 move routes with levels of intensity from 70 to $100 \%$ of the climbing grade performance. Participants trained 6 days a week (Friday being a rest day), with each session lasting from 1.5 to 2 hours. The training plan was standardized, elaborated, supervised and monitored by Eva López, climbing coach. When a training session included dead-hangs, these were performed at the beginning, and a 15-min rest would be included before the climbing contents. On weekends climbing took place on rock routes, 10 to $25 \mathrm{~m}$ long and with difficulties depending on everyone's characteristics. The participants were told perform do 1-2 routes for a warm-up and 1-2 routes close to their maximum level. This was checked via the daily feedback that was monitored during the whole length of the study. 
Participants were not allowed to perform any additional training not prescribed for this study.

\section{Statistical Analysis}

Data were analysed using the Statistical Package for Social Sciences (SPSS; version 17) and Microsoft Excel 2007. Descriptive statistics (mean and standard deviation) were calculated for age, years of training experience, body height, body mass, sport activity in the last 6 months as well as for the strength and endurance test results. The repeated measures ANOVA with Bonferroni adjustment was performed to estimate the intraand intergroup differences. Pearson's correlation coefficients were also estimated to determine the association between variables. The effect size (ES) was calculated to evaluate intragroup changes (Hedges and Olkin, 1985) with the formula $g=$ (M1 - M2) / SDpooled, where M1 was the average for the initial test, M2 the average for the posttraining test and SDpooled was the pooled standard deviation of the corresponding standard deviations. An ES $<0.25$ was defined as trivial, 0.25 to 0.50 small, $0.50-1$ moderate and $>1$ large, according to the scale proposed by Rhea (2004) for strength training interventions on well-trained athletes. The differences between averages and correlations were considered significant when the probability of error was $\leq 5 \%$. All statistical analyses were restricted to the subgroup of athletes that completed the study $(n=26)$.

\section{Results}

The initial, pre-training strength and endurance tests did not show significant differences between groups. There were significant increases in grip endurance for IntHangs athletes after $4(25.2 \%, p=0.004)$ and 8 weeks of training $(45 \%, p=0.002)$, as well as for MaxHangs athletes after 8 weeks of training $(34.1 \%, p=0.010)$ and from the fourth to the eighth week $(23.9 \%, p=0.038)$. Although IntHangs and MaxHangs climbers experienced an improvement in endurance after 8 weeks that was nearly 8 and 5 times that of Max_IntHangs, respectively (Table 2), there was no statistically significant difference among groups in ET2 and ET3 $(p>0.05)$. Significant positive correlations between ET1 and climbing ability (controlling for body mass) were found before training $(r=0.62, p$ $<0.001)$, as well as between strength and endurance after 4 and 8 weeks of training $(r=0.83$ and $0.84, p<0.001$, respectively) and between grip strength and grip endurance before training $(\mathrm{r}=$ $0.83 ; p<0.001)$.

\begin{tabular}{|c|c|c|c|c|c|}
\hline \multirow{2}{*}{\multicolumn{6}{|c|}{ Descriptive statistics by group (mean $\pm S D)$. }} \\
\hline & & & & & \\
\hline Group & Age (years) & $\begin{array}{l}\text { Body height } \\
\quad(\mathrm{cm})\end{array}$ & Body mass (kg) & $\begin{array}{l}\text { Climbing } \\
\text { experience } \\
\text { (years) }\end{array}$ & $\begin{array}{l}\text { Mean climbing } \\
\quad \text { ability } \\
\text { (French scale) }\end{array}$ \\
\hline $\begin{array}{l}\text { MaxHangs } \\
(\mathrm{n}=11)\end{array}$ & $33.91 \pm 7.00$ & $171.3 \pm 7.41$ & $67.04 \pm 8.90$ & $14.27 \pm 6.34$ & $8 a$ \\
\hline $\begin{array}{l}\text { Max_IntHangs } \\
(\mathrm{n}=7)\end{array}$ & $31.11 \pm 5.30$ & $172.6 \pm 9.31$ & $63.30 \pm 9.93$ & $10.44 \pm 5.75$ & $8 a$ \\
\hline $\begin{array}{l}\text { IntHangs } \\
(\mathrm{n}=8)\end{array}$ & $30.13 \pm 5.77$ & $171.0 \pm 5.57$ & $66.51 \pm 6.90$ & $11.19 \pm 6.14$ & $7 \mathrm{c}+$ \\
\hline \multicolumn{6}{|c|}{$\begin{array}{c}\text { Climbing ability = the best redpoint ascent achieved in the past } 6 \text { months } \\
\text { ("redpoint" means leading a sport route after having practiced the route beforehand). } \\
\text { MaxHangs = } 4 \text { weeks of maximal dead-hangs with maximum added weight and then } 4 \text { weeks } \\
\text { of maximal dead-hangs on the minimum edge depth; Max_IntHangs = } 4 \text { weeks of maximal } \\
\text { dead-hangs with maximum added weight and then } 4 \text { weeks of intermittent } \\
\text { dead-hangs on minimum edge depth; IntHangs = } 8 \text { weeks of intermittent } \\
\text { dead-hangs on minimum edge depth. }\end{array}$} \\
\hline
\end{tabular}




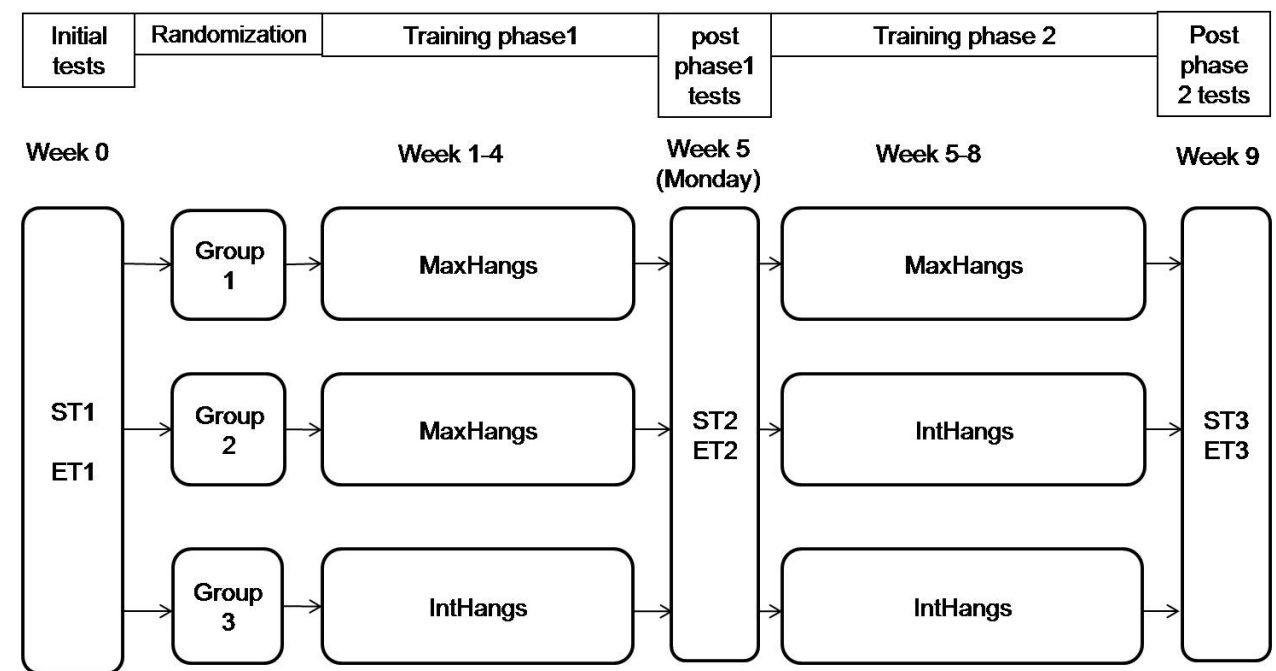

Figure 1

Training and test programs.

MaxHangs $=4$ weeks of maximal dead-hangs with maximum added weight and then 4 weeks of maximal dead-hangs on the minimum edge depth; Max_IntHangs = 4 weeks of maximal dead-hangs with maximum added weight and then 4 weeks of intermittent dead-hangs on minimum edge depth; IntHangs $=8$ weeks of intermittent dead-hangs on minimum edge depth.; $S T 1=$ initial strength test; $E T 1=$ initial endurance test;

$S T 2=$ strength test after 4 weeks of training; ET2 = endurance test after 4 weeks of training; ST3 = strength test after 8 weeks of training; ET3 = endurance test after 8 weeks of training

Figure 2

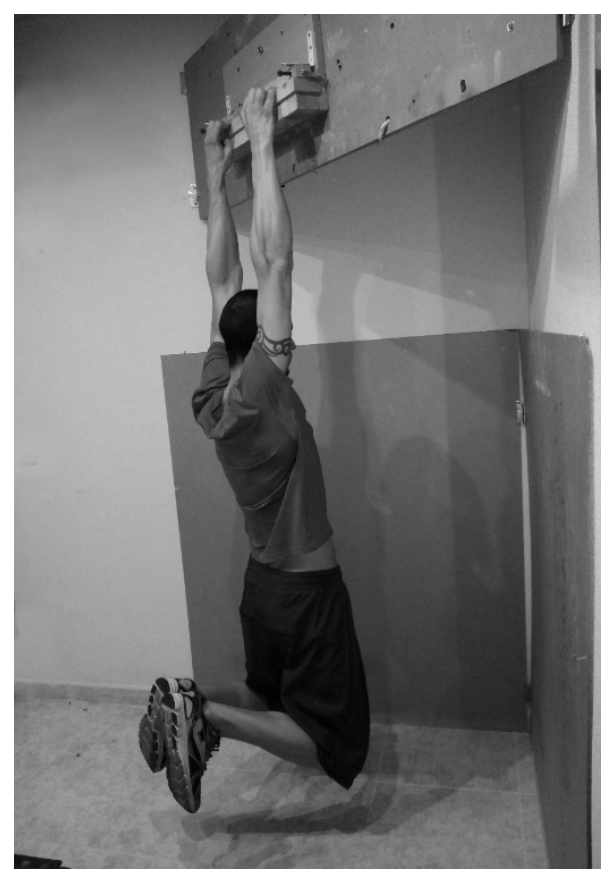

Body position for the endurance test 


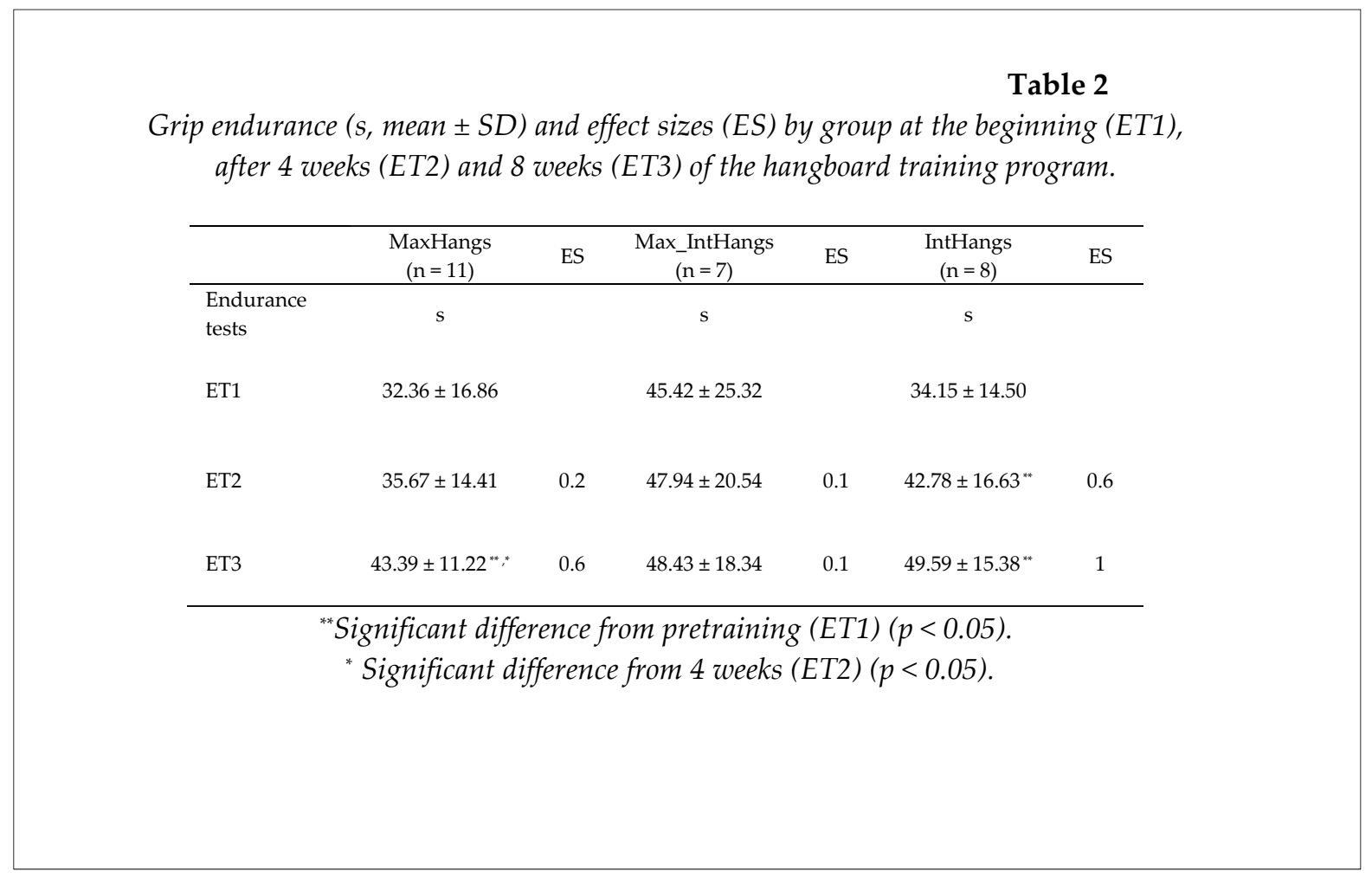

\section{Discussion}

To the best of our knowledge, this is the first study that has compared the effects of three dead-hang training programs on grip endurance in expert rock climbers (mean climbing ability of $7 \mathrm{c}+/ 8 \mathrm{a}$, ranging from $7 \mathrm{a}$ to $8 \mathrm{c}+$ or intermediate to elite level according to Draper et al., 2015). The most relevant results include significant gains in the IntHangs group after 4 and 8 weeks. This group presented the largest improvement in grip endurance in both ET2 and ET3, although these differences were not statistically significant among groups, probably due to the small sample size. Another interesting result is the fact that the MaxHangs group increased significantly grip endurance after 8 weeks of training using a purely strength method. Lastly, the significant relationship found between the endurance test and climbing ability before training supports the importance of grip endurance in climbing.

Even though dead-hangs are extensively used by climbers to improve their finger strength and endurance, we are not aware of any studies comparing the effects of different strength and endurance dead-hang training methods similar to ours with duration of 8 weeks in sport climbers. Yet, there are examples of research in this area using dynamic methods, showing that a high number of repetitions with incomplete rest between them, similar to our IntHangs method, is more suitable for the development of endurance than a method where the intensity is maximal and the repetitions are fewer, analogous to the MaxHangs method (Campos et al., 2002; McGee et al., 1992; Robinson et al., 1995). In the only study to date that has used dead-hang training, Medernach et al. (2015) investigated the effects of 4 weeks of IntHangs on grip endurance in highly advanced boulderers and they found a slightly lower mean increase range in maximum hanging time of 5.5 to $6.7 \mathrm{~s}$, compared to 6.5 to $10.76 \mathrm{~s}$ obtained in this study by the IntHangs group after 4 weeks of training. The reasons behind this fact might be the different hang-to-rest ratio used in the mentioned study, with a hanging time of $8 \mathrm{~s}$ and a rest time of $4 \mathrm{~s}$, combined with a lower ability to improve endurance levels in boulderers in contrast to sport climbers, due to their more explosive profile (Fanchini et al., 2013; Laffaye et., 2014). Other studies have evaluated the effects of high-intensity isometric grip endurance training and found increases in endurance comparable to the $45 \%$ obtained in our study, although in a shorter timespan of 4 to 5 weeks. McDonagh and 
Davies (1984) trained the elbow flexors and finger flexors respectively using a dynamometer and observed a $40 \%$ rise in the fatigue index in untrained men after a training routine of 3 to 5 sets of 10 maximal contractions with $20 \mathrm{~s}$ rest between sets. Similar figures of a $40 \%$ increase in time until exhaustion $(p<0.05)$ were registered by Usaj (2001) after a program of 30 to $75 \mathrm{~s}$ sets of continuous isometric contractions at $27 \% \mathrm{MVC}$ in untrained volunteers. The faster rate of change in these studies could be due to the participants lacking prior training experience (Peterson et al., 2004).

The physiological explanation for this rise in grip endurance, or more specifically in the ability to maintain a high level of strength in the IntHangs group, can reside in: enhanced glycogen and phosphagen storage (Bertuzzi et al., 2007); increased maximum strength, probably via hypertrophy as a result of the use of submaximal loads and incomplete pauses (Robinson et al., 1995; Usaj, 2001); improved oxygen delivery, perfusion and uptake within the muscle that enhances skeletal muscle oxidative capacity (Ferguson and Brown, 1997; Fryer et al., 2014, 2016; Thompson et al., 2014); changes in lactic acid buffering and glycolytic activity (McGee et al., 1992; Robinson et al., 1995) and higher efficiency for dealing with submaximal loads along with improved muscle recruitment patterns (Hawley and Stepto, 2001; Komi et al., 1978).

It is worth noting that the MaxHangs group, that trained for strength, experienced a $34 \%$ improvement in endurance that, although lower than the $45 \%$ obtained by the IntHangs group that trained for endurance, can still be considered significant for a group that did not use endurance methods. This result exceeds the $17 \%$ obtained by López-Rivera and González-Badillo (2012) in the study that assessed the effects of the MaxHangs method for the first time. One reason for this could be the lower average performance level of the participants in the present work (mean climbing ability $7 \mathrm{c}+/ 8 \mathrm{a}$, compared to $8 \mathrm{a}+/ \mathrm{b}$ in the previous study). We have not found any additional studies that measured the effects of strength training using dead-hangs on grip endurance in climbing, but more general isometric training works have registered a $20 \%$ increment in MIF and a $29 \%$ rise in the maintenance of a static load at $60 \%$ of MVC after a 12-week program consisting of 3 to $5 \mathrm{~s}$ contractions (Komi et al., 1978). The suggestion of a strong link between both qualities is supported by the high positive correlation found between the ST1 and ET1 before training and the ST2 and ET2, as well as between the ST3 and ET3. With respect to this, López-Rivera and GonzálezBadillo (2012) also observed a positive correlation $(\mathrm{r}=0.76 ; p<0.05)$ between changes in strength and changes in endurance in elite climbers after 8 weeks of training. A possible physiological explanation of enhancement of grip endurance after training for strength with maximum intensity is that the improved isometric strength enables to bear the load (body mass hanging off an edge) with a reduced proportion of Type II motor units which may result in less lactate accumulation, allowing to extend the time to fatigue for the same load (Hickson et al., 1988; Marcinik et al., 1991). In light of these results, we put forward the suggestion that training strength using dead-hangs, first with added weight and then with small edges, not only develops maximum strength, but also has a notable effect on small-holds grip endurance in climbers.

On the other hand, we expected larger gains in the MaxHangs-IntHangs group, given that 4 weeks of strength training had already yielded a positive effect on grip endurance in the study of López-Rivera and González-Badillo (2012), not to mention that the IntHangs method in weeks 5 to 8 resulted in better endurance in the IntHangs group. Quite surprisingly, the former attained just a $1 \%$ gain after 4 weeks of endurance training (from ET2 to ET3), in contrast with 25\% for the latter after the first 4 weeks, at the ET2. Some possible explanations are a) this combination of exercises provoked excessive fatigue that compromised both strength and endurance development, $b$ ) the probability of a "ceiling effect" that could limit percent of change, given this group's ET1 score was the highest of the three, c) this group presented a high standard deviation and registered more drop-outs, which could have had high impact on the data. The reasons for these drop-outs, though varied, were unrelated to the course of the present study.

Finally, this is the first work to ascertain the importance of endurance in climbing through tests and exercises congruent with high-intensity climbing: maximum hanging duration on small 
holds. The only study we found that involved a specific dead-hang exercise in this context (Baláš et al., 2012) used a deeper edge than ours $(22 \mathrm{~mm}$ or one phalange in contrast to $11 \mathrm{~mm}$ or about one-half of the distal phalange) and obtained a correlation between hanging time and climbing ability of $\mathrm{r}=0.80(p<0.005)$ that is higher than $\mathrm{r}=$ $0.62(p<0.001)$ from the present study. The greater number of participants $(n=209)$ or precisely the difference in edge depth can be among the reasons for this discrepancy.

\section{Conclusions}

The main conclusion of the present study is that the IntHangs program seems to be highly effective for improving grip endurance over duration of 8 weeks. Nonetheless, 4 weeks of weighted dead-hangs on a medium-sized edge followed by 4 weeks of unweighted dead-hangs on the smallest possible edge also lead to an improvement in small-edge endurance, probably due to greater maximum strength as suggested by the high correlation found between changes in strength and changes in endurance after training.

Some limitations should be acknowledged. First, the small sample size of the groups may have contributed to insufficient statistical power for detection of differences among the groups. Moreover, the greater number of drop-outs in the Max_IntHangs and IntHangs groups compared to the MaxHangs group, from an already small number of subjects, could detract from a thorough statistical analysis. However, the difficulty of accessing to advanced and elite sport climbers willing to undertake a 10-week experimental study should be taken in consideration. Future studies should include a larger sample size and might be focused on a narrower range of climbing levels than ours. Nonetheless, given the relevance of grip endurance for climbing performance found by this and previous research, we believe that our work contributes to fill a void that exists in the investigation areas of training methodology and periodization in sport climbing (Watts, 2004), providing climbers and coaches with tools for better individualization of finger training. For instance, the MaxHangs method can be chosen to increase strength along with endurance, while IntHangs to prioritise endurance. Given the importance of both strength and endurance in climbing, in other instances the previously cited methods can be chained or combined during a training cycle.

\section{References}

Amca AM, Vigouroux L, Aritan S, Berton E. Effect of hold depth and grip technique on maximal finger forces in rock climbing. J Sports Sci, 2012; 30(7): 37-41

Baláš J, Pecha O, Martin AJ, Cochrane D. Hand-arm strength and endurance as predictors of climbing performance. Eur J Sport Sci, 2012; 12(1): 16-25

Bertuzzi RCDM, Franchini E, Kokubun E, Kiss MAPDM. Energy system contributions in indoor rock climbing. Eur J Appl Physiol, 2007; 101(3): 293-300

Campos GER, Luecke TJ, Wendeln HK, Toma K, Hagerman FC, Murray TF, Ragg KE, Ratamess NA, Kraemer WJ, Staron RS. Muscular adaptations in response to three different resistance-training regimens: Specificity of repetition maximum training zones. Eur J Appl Physiol, 2002; 88(1-2): 50-60

Demura S, Nakada M. Relationships between force and muscle oxygenation kinetics during sustained static gripping using a progressive workload. J Physiol Anthropol, 2009; 28(3): 109-114

Draper N, Giles D, Schöffl V, Fuss FK, Watts PB, Wolf P, Balás J, España-Romero V, Gonzalez GB, Fryer S, Fanchini M, Vigouroux L, Seifert L, Donath L, Spoerri M, Bonetti K, Phillips K, Stöcker U, BourassaMoreau F, Garrido I, Drum S, Beekmeyer S, Ziltener JL, Taylor N, Beeretz I, Mally F, Amca AM, Linhart C, Abreu E. Comparative grading scales, statistical analyses, climber descriptors and ability grouping: International Rock Climbing Research Association position statement. Sport Technology. 2015; 8(3-4):88-94

Ebben WP, Kindler, AG, Chirdon, KA, Jenkins NC, Polichnowski, AJ, Ng AV. The effect of high-load vs 
high-repetition training on endurance performance. J Strength Cond Res, 2004; 18(3): 513-517

Fanchini M, Violette F, Impellizzeri F, Maffiuletti N. Differences in climbing-specific strength between boulder and lead rock climbers. J Strength Cond Res; 2013, 27(2): 310-314

Ferguson RA, Brown MD. Arterial blood pressure and forearm vascular conductance responses to sustained and rhythmic isometric exercise and arterial occlusion in trained rock climbers and untrained sedentary subjects. Eur J Appl Physiol O, 1997; 76(2): 174-180

Fryer S, Stoner L, Lucero A, Witter T, Scarrott C, Dickson T, Cole M, Draper N. Haemodynamic Kinetics and Intermittent Finger Flexor Performance in Rock Climbers. Int J Sports Med, 2014; 36(2): 137-142

Fryer S, Stoner L, Stone K, Giles D, Sveen J, Garrido I, España-Romero V. Forearm muscle oxidative capacity index predicts sport rock-climbing performance. Eur J Appl Physiol, 2016; 116(8): 1479-1484

Hawley JA, Stepto NK. Adaptations to training in endurance cyclists: implications for performance. Sports Med, 2001; 31(7): 511-520

Hickson RC, Dvorak BA, Gorostiaga EM, Kurowski TT, Foster C. Potential for strength and endurance training to amplify endurance performance. J Appl Physiol, 1988; 65(5): 2285-2290

Iglesias E, Boullosa DA, Dopico X, Carballeira E. Analysis of factors that influence the maximum number of repetitions in two upper-body resistance exercises: curl bíceps and bench press. J Strength Cond Res, 2010; 24(6): 1566-1572

Komi PV, Viitasalo JT, Rauramaa R, Vihko V. Effect of isometric strength training on mechanical, electrical, and metabolic aspects of muscle function. Eur J Appl Physiol, 1978; 40(1): 45-55

Laffaye G, Collin JM, Levernier G, Padulo J. Upper-limb Power Test in Rock-climbing Personal. Int J Sport Med 2014; 35(8): 670-675

López-Rivera E, González-Badillo JJ. The effects of two maximum grip strength training methods using the same effort duration and different edge depth on grip endurance in elite climbers. Sports Technology, 2012; 5(3-4): 1-11

MacLeod D, Sutherland DL, Buntin L, Whitaker A, Aitchison T, Watt I, Bradley J, Grant S. Physiological determinants of climbing-specific finger endurance and sport rock climbing performance. J Sports Sci, 2007; 25(12): 1433-1443

Magiera A, Roczniok R, Maszczyk A, Czuba M, Kantyka J, Kurek P. The structure of performance of a sport rock climber. J Hum Kinet. 2013; 36: 107-17

Marcinik E, Potts J, Schlaback G, Dawson P, Hurley B. Effects of strength training on lactate threshold and endurance performance. Med Sci Sport Exer, 1991; 23(6): 739-743

McDonagh MJ, Davies CT. Adaptive response of mammalian skeletal muscle to exercise with high loads. Eur J Appl Physiol O, 1984; 52(2): 139-155

McGee D, Jessee TC, Stone MH, Blessing D. Leg and Hip Endurance Adaptations to Three Weight-training Programs. J Strength Cond Res, 1992; 6(2): 92-95

Medernach JPJ, Kleinöder H, Lötzerich HHH. Fingerboard in competitive bouldering: training effects on grip strength and endurance. J Strength Cond Res, 2015; 29(8): 2286-2295

Peterson MD, Rhea MR, Alvar BA. Maximizing strength development in athletes: a meta-analysis to determine the dose-response relationship. J Strength Cond Res, 2004; 18(2): 377-382

Philippe M, Wegst D, Müller T, Raschner C, Burtscher M. Climbing-specific finger flexor performance and forearm muscle oxygenation in elite male and female sport climbers. Eur J Appl Physiol, 2012; 112(8): 2839-2847

Pucci AR, Griffin L, Cafarelli E. Maximal motor unit firing rates during isometric resistance training in men. Exp Physiol, 2006; 91(1): 171-178

Rhea MR. Determining the magnitude of treatment effects in strength training research through the use of the effect size. J Strength Cond Res, 2004; 18(4): 918-920

Robinson JM, Stone MH, Johnson RL, Penland CM, Warren BJ, Lewis RD. Effect of different weight training exercise/rest intervals on strength, power, and high intensity exercise endurance. J Strength Cond Res, 
1995; 9(4): 216-221

Schweizer A. Biomechanical properties of the grip position in rock climbers. J Biomech, 2001; 34(2): 217-223

Schweizer A, Hudek R. Kinetics of Crimp and Slope Grip in Rock Climbing. J Biomech, 2011; 27(2): 116-121

Tanaka H, Costill DL, Thomas R, Fink WJ, Widrick JJ. Dry-land resistance training for competitive swimming. Med Sci Sport Exer, 1993; 25(8): 952-959

Tomlin DL, Wenger HA. The relationship between aerobic fitness and recovery from high intensity intermittent exercise. Sports Med, 2001; 31(1): 1-11

Usaj A. The endurance training effect on the oxygenation status of an isometrically contracted forearm muscle. Pflug Arch Eur J Phy, 2001; 442(Suppl 1): R155-R156

Vigouroux L, Quaine F. Fingertip force and electromyography of finger flexor muscles during a prolonged intermittent exercise in elite climbers and sedentary individuals. J Sports Sci, 2006; 24(2): 181-186

Watts P. Physiology of difficult rock climbing. Eur J Appl Physiol, 2004; 91(4): 361-372

White DJ, Olsen PD. A time motion analysis of bouldering style competitive rock climbing. J Strength Cond Res, 2010; 24(5): 1356-1360

\section{Corresponding author:}

\section{Eva López-Rivera,}

Faculty of Sport Sciences, Castilla La-Mancha University, Avda. Carlos III, 45004 Toledo, Spain.

Phone number: 9252688 00, Fax number: 925268800.

Email: evalopriv@gmail.com 